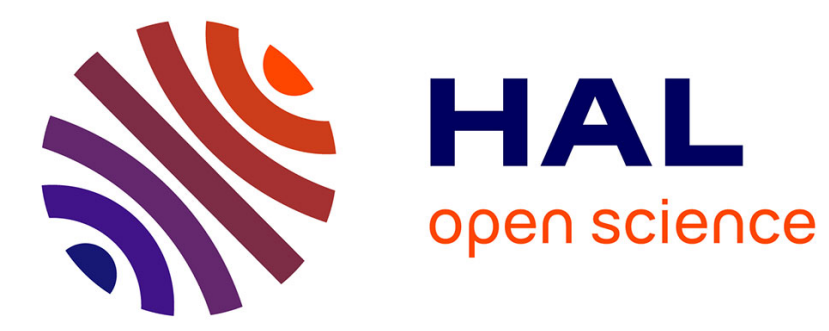

\title{
Cooperative self Organization of agents for optimization : the electrical wiring example
}

Stéphanie Combettes, Pierre Glize, Thomas Sontheimer, Sylvain Rougemaille

\section{To cite this version:}

Stéphanie Combettes, Pierre Glize, Thomas Sontheimer, Sylvain Rougemaille. Cooperative self Organization of agents for optimization: the electrical wiring example. 5th International Conference on Agents and Artificial Intelligence (ICAART 2013), Feb 2013, Barcelona, Spain. pp. $482-485$. hal-01282035

\section{HAL Id: hal-01282035 \\ https://hal.science/hal-01282035}

Submitted on 3 Mar 2016

HAL is a multi-disciplinary open access archive for the deposit and dissemination of scientific research documents, whether they are published or not. The documents may come from teaching and research institutions in France or abroad, or from public or private research centers.
L'archive ouverte pluridisciplinaire HAL, est destinée au dépôt et à la diffusion de documents scientifiques de niveau recherche, publiés ou non, émanant des établissements d'enseignement et de recherche français ou étrangers, des laboratoires publics ou privés. 


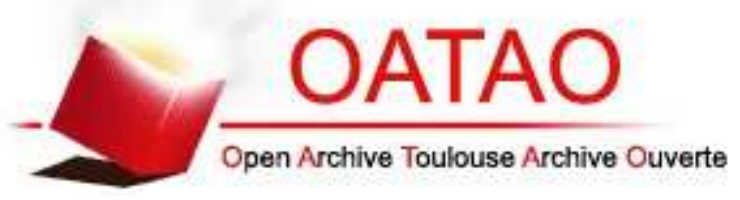

\section{Open Archive TOULOUSE Archive Ouverte (OATAO)}

OATAO is an open access repository that collects the work of Toulouse researchers and makes it freely available over the web where possible.

This is an author-deposited version published in : http://oatao.univ-toulouse.fr/ Eprints ID : 12365

The contribution was presented at:

http://www.icaart.org/? $\mathrm{y}=2013$

To cite this version : Combettes, Stéphanie and Glize, Pierre and Sontheimer, Thomas and Rougemaille, Sylvain Cooperative self Organization of agents for optimization : the electrical wiring example. (2013) In: $5^{\text {th }}$ International Conference on Agents and Artificial Intelligence (ICAART 2013), 15 February 2013 - 18 February 2013 (Barcelona, Spain).

Any correspondance concerning this service should be sent to the repository administrator: staff-oatao@listes-diff.inp-toulouse.fr 


\title{
Cooperative self-organization of agents for optimization : the electrical wiring example
}

\author{
Stéphanie Combettes ${ }^{1}$, Pierre Glize ${ }^{1}$,Thomas Sontheimer ${ }^{2}$ and Sylvain Rougemaille ${ }^{2}$ \\ ${ }^{1}$ Institut de Recherche en Informatique de Toulouse, Université Paul Sabatier - Toulouse III, France \\ ${ }^{2}$ Upetec, Ramonville-Saint-Agne, France \\ $\{$ stephanie.combettes, pierre.glize\}@irit.fr, \{thomas.sontheimer,sylvain.rougemaille\}@upetec.fr
}

\begin{abstract}
Keywords: Emergent optimization, cooperative self-organization, autonomous local decision
Abstract: In aircrafts, densifying electrical systems and oversizing cables in order to respect constraints induce a useless increase in cable weight. This increase leads to additional costs of operation and to an unnecessary pollution during the plane operating life. In this paper we address optimization of harness weight which is a monoobjective problem with manifold and interdependent constraints. To solve this problem, we use a multi-agent approach based on the cooperative self-organization of agents. Performances obtained by the 'Smart Harness Optimizer' software that we have developed are promising for problems considered by the experts as being very difficult. In this article, we expose the method used to solve this Constraint Optimization Problem. Then we describe the agentification of this problem and the steps of the resolution. Finally we give results on typical cases and analyze them.
\end{abstract}

\section{INTRODUCTION}

In the prospects of more electrical aircraft, functions that used pneumatics or hydraulics systems are now realized by electrical systems. Moreover cabins offer to passengers a more important space to improve their comfort. Consequently, the electrical system, also called electrical harnesses within aircrafts becomes denser. An electrical harness is an assembly of cables connecting together electrical appliances. Within an aircraft, the route of harnesses and the grouping of cables into predefined harnesses obey rules that become more difficult to respect because of this densification. Thus margins initially taken by the designer ensure a good sizing of cables. More precisely cable diameter is over evaluated for security reasons but however it also increases aircraft weight. This increase leads to additional costs of operation and to an unnecessary pollution during the plane operating life.

Optimizing the sizing of electrical harnesses is a real opportunity, which while ensuring the respect of electrical rules leads to reduce the harness weight.

However this issue is not straightforward because of the numerous and interdependent constraints to respect. Moreover the number of elements present in an electrical system may be huge, which also complicates the resolution of the problem.
In this paper we show how this optimization problem may be solved without the solution search is guided by the knowledge of the global state, but with local objectives.

To this aim, part 2 presents the problem to solve by describing the architecture of the electrical system and the constraints to solve. Part 3 gives an overview of the solving methods and presents the Adaptive Multi-Agent System (AMAS) approach, a method of emergent resolution. Part 4 applies this approach to the optimization of harness weight. Part 5 presents the results and analyzes them.

\section{DESCRIPTION OF THE PROBLEM}

Minimizing the harness weight consists actually in optimizing the gauge of cables. A gauge is a discrete measure of a cable cross-section; increasing gauges gives decreasing diameters and so lighter cables. We rapidly describe the electrical system as being mainly composed of harnesses, functional links, cables and wires of cables (we will give more details later in this section). A lot of dependencies exist between the different components of this electrical system and their characteristics depend on four fight phases (landing, 
parking, flying and taking off). Additionally some environmental, electrical and thermic constraints must be respected. As this work is a part of a project, it has been simplified to adapt it to the project duration, more precisely not all the constraints have been taken in which this problem has been defined. Thus we only focus on the electrical and thermic constraints.

In an aircraft several functional links are carried out by hundreds of cables constituting tens of harnesses. Selecting a maximal gauge (minimal diameter) for a cable does not mean respecting electrical constraints, so the compromise to solve is to select the smallest gauge while respecting all the constraints. This is a combinatorial optimization problem requiring to explore a huge search space of solutions. In the following we describe in more details first the architecture of an electrical system, second the constraints to consider and third we give a formalization of the problem.

\subsection{Architecture of the Electrical System}

The electrical distribution consists in bringing energy from production hearts towards different consumer systems. The design of the electrical system in an aircraft consists in dealing with the topology of the aircraft, the pressure and non-pressure areas, the location of the electrical devices within the aircraft and the possible routes for harnesses. For security reasons some equipment connections must be duplicated and have different routes. The grouping of cables into an harness also has to respond to some constraints (such as temperature or overheating). As it may be understood, the design of an electrical system is a hard task to fulfill and in addition the structure of an harness is an aggregation of several elements.

We shall now develop the architecture of an electrical harness which is twofold: a physical and a functional points of views. According to the physical point of view, equipments are connected by wires, themselves aggregated into cables to reduce both the weight and the cost of the cladding and shield. Cables are themselves gathered within a harness. A harness forms an arborescence that is defined according to the routes reserved in the aircraft structure for passing through the electrical distribution. The unit element of this arborescence is a branch. It corresponds to a space located between two nodes, where environmental conditions of temperature and pressure are homogeneous. Figure 1 shows a schematic representation of the physical view of a harness.

According to the functional point of view, a

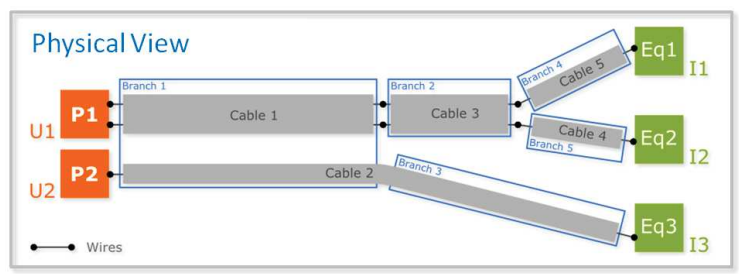

Figure 1: A physical view of a harness.

production system is connected to consumer equipments via links going through harnesses. A link is represented by a succession of wires. Figure 2 shows a schematic representation of the functional view of a harness.

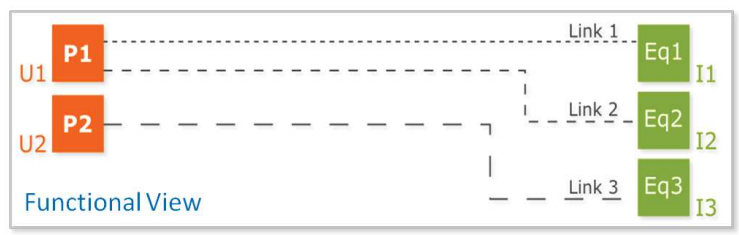

Figure 2: A functional view of a harness.

To summarize, a harness contains physically cables and functionally links. Wires are at the intersection of cables and links, as they are related to these two elements.

\subsection{The Constraints}

The design of an electrical system has to take into account numerous, manifold and interdependent constraints to enable a secure functioning of the aircraft during its operation life. In our problem we narrow the set of constraints to the electrical and thermic ones that we now detail by explaining to which elements they have to be applied and the consequences of their non-respect.

A maximal voltage drop is associated to each link, which must not be exceeded at risk of dysfunction of the powered system.

A maximal temperature and a maximal overheating are associated to each cable, which must not be exceeded at risk of melt. Moreover, all the wires gathered in a cable must have the same gauge.

In addition to being interdependent, all of these constraints have to be checked for all the flight phases (landing, parking, flying and taking off) as the value of each constraint depends on the flight phase. For instance considering the harness sizing rules, decreasing a cable diameter means an increase of its 
temperature and its voltage drop increase, and vice versa.

A plane may contain up to one thousand harnesses, each of them may contain tens of cables having themselves up to four wires. So there are about fifty thousand interdependent variables.

Considering the voltage drop, overheating and temperature constraints and our objective of minimizing the weight of the electrical system, this optimization problem to solve is multi-constrained, monoobjective and NP complete.

\subsection{Formalization of the Harness Weight Optimization}

Any optimization problem may be characterized using the CSP formalism. A CSP is a triplet $\langle X, D, C\rangle$ such as $X=\left\{x_{1}, \ldots, x_{n}\right\}$ is the set of variables to instantiate. $D=\left\{D_{1}, \ldots, D_{m}\right\}$ is the set of domains. Each variable $x_{i}$ is related to a domain of value. $C=$ $\left\{c_{1}, \ldots, c_{k}\right\}$ is the set of constraints, which are relations between some variables from $X$ that constrain the values the variables can be simultaneously instantiated to. Considering the harness weight optimization, this problem is described as follows.

- A set of wires $W=w_{1}, \ldots, w_{m}$ with $m \in \mathbb{N}$;

- The sets of domains are $\mathbb{R}^{+}$for a range of diameter (continuous values) and a set of gauges $G=g_{1}, \ldots, g_{10}$ (discrete values);

- Constraints. Let be the following sets :

- A set of links $L=l_{1}, \ldots, l_{n}$ with $n \in \mathbb{N}$;

- A set of connections $O=o_{1}, \ldots, o_{j}$ with $j \in \mathbb{N}$;

- A set of cables $C=c_{1}, \ldots, c_{p}$ with $p \in \mathbb{N}$;

- $\forall l_{i} \in L$, VoltageDrop < MaxVoltageDrop (each link has to check that the voltage drop between ends of the wires that form it is less than the maximal authorized voltage drop);

- $\forall c_{i} \in C$, Temperature $<$ MaxTemperature and $\forall c_{i} \in C$, Overheating $<$ MaxOverheating (each cable has to check the temperature and overheating constraints);

- Let be a function BelongTo $: W \longmapsto C$ giving the cable containing the considered wire.

Let be another function Gauge $: W \longmapsto G$, giving the gauge value of the considered wire.

Let $w_{k} \in W \mid$ BelongTo $\left(w_{k}\right)=c_{j}$, then $\forall w_{i} \in$ $W$ and BelongTo $\left(w_{i}\right)=c_{j}$, Gauge $\left(w_{k}, t\right)=$ Gauge $\left(w_{i}, t\right)$ (The wire diameter has to be identical to those of wires belonging to the same cable);
- Let be a function ConnectedTo $: W \longmapsto O$ giving the connection of the considered wire. Let be a function Voltage $: W \longmapsto \mathbb{R}$ giving the voltage of the considered wire.

$\forall w_{i} \in W \mid$ ConnectedTo $\left(w_{i}\right)=o_{j}$, $\sum \operatorname{Voltage}\left(w_{i}\right)=0$ (each connection connecting several wires belonging to a same link has to balance the charge between wires).

The problem to solve is :

$$
S=\operatorname{Min}\left(\sum_{i=1 . . m} \operatorname{Weight}\left(w_{i}\right)\right)
$$

with Weight $: W \longmapsto \mathbb{R}^{+}$be a function giving the weight of the wire $w_{i}$.

\section{WEIGHT OPTIMIZATION PROBLEM SOLVING}

Different formalisms have been developed for solving complex optimization problems under constraints, and among them, the most widely studied is the Constraint Optimization Problem (COP) formalism. In this formalism, problems are translated in a given framework. Thus a set of variables (problem entities) must be assigned a value of a given domain in order to minimize or maximize an objective function. Solving such problems consists in exploring the search space and finding the best assignment to those variables.

\subsection{Complexities and Limits of Optimization Problems}

The complexity of optimization problems is due to the heterogeneity and diversity of the participating actors, their evolving constraints or the interdependency of the involved parameters making a global comprehension of the problem difficult or even impossible.

The current applications have a growing complexity and are confronted to unpredictable and changing events that produce dynamics in the system. So, in some domains translating the problem into a COP formalism is impossible as the process of resolution depends on the experts knowledge and experience. Moreover, those current applications have an important number of elements and constraints to be respected that implies a combinatorial explosion of the search space. Finding the optimal solution becomes difficult even impossible or requires prohibitive computation times because of this large number of variables (Talbi, 2009), (Yokoo et al., 1998).

To solve COP, several methods have been developed among them the Meta-heuristics, an approximate one, which we will focus on. 


\subsection{Overview of the Meta-heuristics}

Several meta-heuristics of local search use different stopping criteria (time, distance to the solution, iteration number) and of displacement. Among these algorithms, efficient but not complete, the most representative are the Tabu search (Glover and Laguna, 1997) and the Simulated Annealing (Kirkpatrick et al., 1983).

Approaches like DBA (Distributed Breakout Algorithm) (Hirayama and Yokoo, 2005) or ERA (Environment, Reactive rules and Agents) (Liu et al., 2002) are based on the same principles consisting in exploring the distribution of the solution. Their drawback is the need of a global optimization based on the objective function which may be not known at the design stage.

Others different algorithms use population-based meta-heuristics. The individuals have local behavior that is quite simply but coordinated to explore the search space. This class of algorithms contains essentially Genetic Algorithms (Holland, 1993), Particle Swarm Optimization (Kennedy and Eberhart, 2001) and Ant Colony (Dorigo and Stützle, 2004).

As each individual of the population in these algorithms has the global description of the problem and potentially the solution, the same difficulty to apply them to the problem of harness weight optimization in aircrafts is met.

The multi-agent technology seems to be pertinent for environments relatively dynamics (constraints and local objectives may evolve) and where the search time is constrained (user waiting time). Moreover disruptions induced by the specification evolution are locally propagated and there is no need to redefine a global objective function and to execute from the beginning the resolution process. For all these reasons, we consider that algorithms enabling emergent search of solutions are more pertinent for our problem.

We propose to use the Adaptive Multi-Agent System (AMAS) theory (Capera et al., 2003) to solve the harness weight optimization. This theory is based on the cooperative self-organization of agents, and whose system aim is to reach the adequate collective function. The self-organization principle consists for agents in satisfying a local criteria thanks to theirs skills and believes, and without being conscious of the global objective to reach. Thus each agent has its own local function and has to cooperate with its neighbor agents in order to enable self-organization (Welcomme et al., 2009).

We shall describe more precisely this approach now.

\subsection{Adaptive Multi-Agent System Approach}

The Adaptive Multi-Agent System (AMAS) approach is based on cooperative self-organization of agents of the system whose aim is to reach the adequate collective function. Cooperation between agents having a local behavior leads to the emergence of the function to the global level, i.e. to the system level. This global behavior is only visible by an observer being outside the system. It is not necessary to define explicitly the global function of the system to solve but to lead agents to make it emerging thanks to their cooperation.

This approach is based on the theorem of the functional adequacy (Glize, 2001) that states that:

For any functionally adequate system, there exists at least one system with cooperative internal medium that fulfills an equivalent function in the same environment.

A cooperative internal medium system is a system where no Non Cooperative Situation (NCS) exists: each agent interacts in a cooperative way with his neighborhood and it is not in SNC. The noncooperation is defined as follow:

$$
\text { NonCoop }=\neg C_{\text {perception }} \vee \neg C_{\text {decision }} \vee \neg C_{\text {action }}
$$

It means that an agent is in a SNC if i) the signal it perceived is ambiguous or not understood, ii) the information perceived does not produce any new decision and iii) the consequences of its actions are not useful to others. An agent may face seven types of SNC during its life cycle (perception, decision, action) described below.

- During the perception phase :

- ambiguity : the agent interprets the perceived signal in several ways;

- incomprehension : the agent does not understand the perceived message.

- During the decision phase:

- unproductiveness : the agent does not produce any conclusions from the perceived information;

- incompetence : the agent is not able to exploit the perceived information.

- During the action phase :

- uselessness : the agent thinks that its action neither will help another agent nor come closer to its own objective;

- conflict : the agent thinks that its action and the one of another agent are antinomic; 
- concurrency: the agent thinks its action and the one of another agent will end up in the same result.

Solving SNCs may be regarded as learning the adequate functionality and represents the critical point of the adaptation process. Thus besides its nominal behavior (i.e. related to its local objective), an agent should be provided a cooperative behavior including detection (and solution) of SNCs, and prevention of SNC occurring.

\subsection{Solving Non Cooperative Situations}

In the AMAS approach, besides its functions of perception, decision and action, the agent is autonomous and owns a local objective that influences its decision function. It may evaluate its non-satisfaction degree according to its current situation. This nonsatisfaction degree also called criticality represents the distance between its current situation and the achievement of its local objective. The more far from its local objective an agent is, the more critical it is. The cooperative attitude of an agent results in the realization of its local goal without increasing, even decreasing, the criticalities of the neighborhood agents. An agent may even deteriorate timely its own situation, if necessary, in order to help a neighbor agent with a too high criticality. So cooperation is the dynamic of the reorganization as it guarantees that the system will reach a functionally adequate state aimed by the designer.

As the description of the AMAS approach was given, we shall apply it to the problem of harness weight optimization in the following section.

\section{APPLYING AMAS TO MINIMIZE HARNESS WEIGHT}

Designing and sizing harness cables by minimizing the weight is a complex problem of combinatorial optimization under constraints. The optimization tools come up against to the exponential increasing of calculation times as the problem becomes more complex. This difficulty narrows the use of these tools for sizing subsets of aircraft wiring and poses the issue of the coherence of the whole.

The AMAS approach leads to a strictly local resolution of the problem. Thus the search space is not totally explored but is guided by the cooperative principle. This paradigm change enables us to free itself from the practical limits met by classical approaches of optimization such as the increase of computation times. Thus cooperation between agents (each having its own local goal) has to make the adequate function emerge, i.e. here minimizing the harness weight.

We now specify the different types of agents composing the system and their behavior.

\subsection{Agents and Local Aim}

The AMAS theory proposes a bottom-up analysis of the problem and leads to its strictly local resolution. From the analysis of the domain and the data model, several Non Cooperative Situation (NCS) were identified. We remind you that these latter represent the inability of the different entities of the data model to solve each identified problem. By distinguishing which entity encounters one of these problems, we identify those whose behavior has to be made cooperative and therefore become a cooperative agent of the system. This agentification phase has enabled us to define the four following types of agents : the Link, Cable, Wire and Connection agents.

The Link agent represents the functional aspects of the electrical system and its local goal is to respect the voltage drop constraints.

The Cable agent represents a cable and its local goal is to uniform the diameter of its wires and to expose a current diameter. It also has to respect temperature and overheating constraints.

The Wire agent represents a wire and binds the functional aspects (links) and the physical ones (cables). Its local objective is to stabilize its diameter (whatever its initial value).

Finally the Connection agent represents a connection point between several wires belonging to a same link. Its local objective is to balance the criticalities of the Wire agents connected to it.

To take into account the four flight phases (landing, parking, flying and taking off), all the Link, Wire and Connection agents were cloned four times, once per flight phase. Only the Cable agents were not cloned. Indeed, a Cable agent is the physical element shared by all the flight phases and it seeks the optimized gauge value satisfying all its Wire agents. The Cable agent is the central element which integrates all the additional constraints of Wire, Connection and Link agents, that are related to the flight phases.

For instance a cable made of three wires is thus represented by a Cable agent having three Wire agents per flight phase, so twelve Wire agents. The Cable agent has to converge towards a common gauge satisfying 
the constraints of all its Wire agents (thus indirectly those of Link and Connection agents). As each wire is in relation with the Link and Connection agents, a modification of its gauge perturbs the voltage drop, etc. and thus implies adaptation of other agents (chain reaction) but also means that other agents may perturb it.

\subsection{Steps of Resolution}

The problem resolution seeks the value of the optimal diameter and is carried out on continuous values. For that purpose, the Wire agents are at the heart of the algorithm. Their own goal is to stabilize their diameter without putting in trouble the Link and Cable agents, i.e. by respecting electro-thermic constraints. Each Wire agent estimates its criticality degree according to its current section. This criticality is locally computed and then communicated to the Connection agent. In the case where no constraint is broken, the Wire agent decreases its criticality by reducing its section.

Each Link agent checks that the voltage drop is respected according to the voltage between ends of Wire agents that form it. As soon as the voltage drop exceeds the maximal authorized one the Link agent is in a Non Cooperative Situation (SNC), and being not able to change itself this incompetence SNC, it informs the Connection agents to whom it is connected. Once it has received the criticality values of the Wire agents to whom it is connected, each Connection agent deduces which Wire agent may act in order to i) solve the Link agent SNC and ii) make the criticality degree decrease.

Each Cable agent checks that no temperature or overheating constraint is violated. Otherwise, incriminated Wire agents (the most critical contained by the Cable agent) are informed and change their current section.

The Wire agents end up determining their optimal sections through this play of modifications (successive increases and decreases) of agents section and through an internal learning mechanism. Those found sections form the basis to agents, for the selection of gauges of wires. This is the succession of changes and the propagation of modification among the agents that leads the system to have the global function that emerges. During this solving phase, the notion of minimizing weight is not explicitly and directly tackled. To show this clearly we will have a focus in the rest of the section on the communication between agents.

\subsection{Focus on Communication between Agents}

Communication between agents is a crucial point that enables them to cooperate. In this aim, we focus here on these exchanges by giving the algorithms of communications of each type of agents. We consider here the first step of the resolution, that is to say the search of the optimal section of cables (continuous part). We remind that the Link agent has to respect the voltage drop constraints.

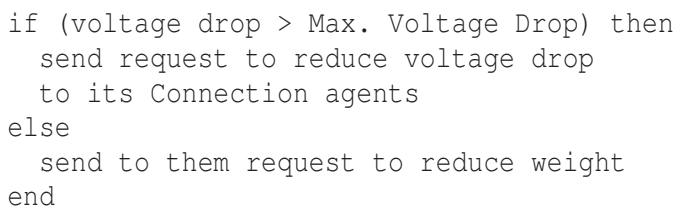

The Connection agent has to balance the criticalities of the Wire agents connected to it.

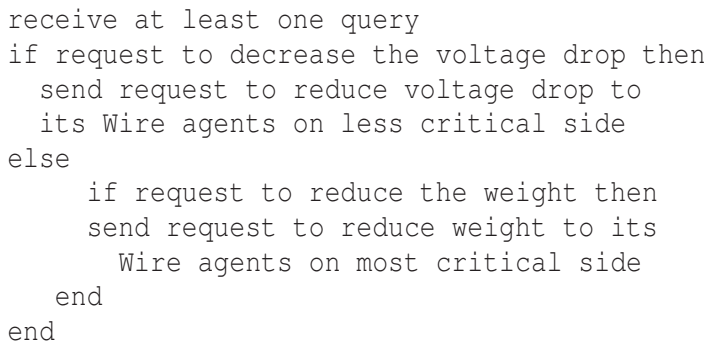

The Wire agent has to stabilize its diameter according to the respect/non-respect of constraints.

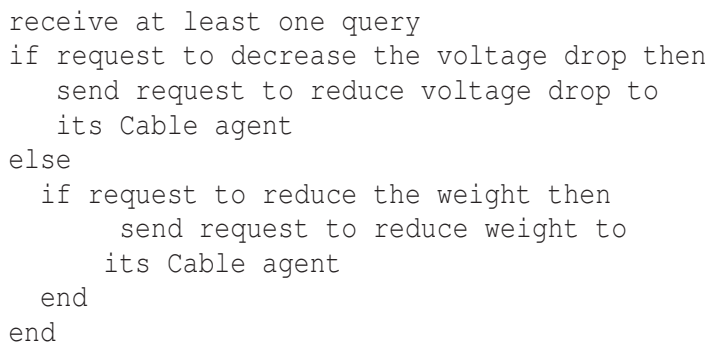

The Cable agent has to respect temperature and overheating constraints and to uniform the diameter of its wires.

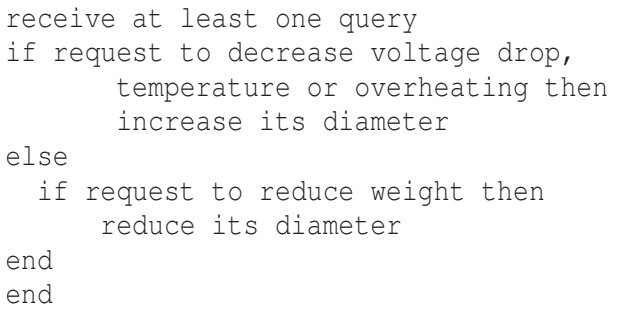

We could first notice that during the resolution time, the weight value is never used or calculated or exchanged between agents. The optimization of the weight is carried out indirectly by increasing or decreasing diameter of cables. This point shows 
that the global objective is not explicitly computed but emerges from the local actions of each agent achieving its own goal.

We second notice that there is no random during the execution of the algorithms as opposed to classical algorithms such as Ants Colony, or Tabu Search. An agent tries only to reduce the degree of its criticality or its neighborhood. We also see in the algorithm that each agent decides at most one action during a cycle.

\section{RESULTS AND ANALYSIS}

We have developed a software platform called Smart Harness Optimizer that implements the AMAS approach using processes of local decision. Its interface may be visualized in Figure 3 and shows the structure of a harness (center) and its elements and characteristics (below).

After describing the test cases, the results are given and then analyzed.

\subsection{Outlines of Test Cases}

Three categories of test cases were used to evaluate the tool developed. They correspond to three electrical systems constituted of respectively 3, 8 and 52 harnesses. Each category comes in several instances where the charge required by equipments was changed. The two first instances are amperage uniformed loaded for all links in all flight phases with 1A, 4A and 20A (10A for the second case). The last instance has amperage modifications depending on flight phases. The case of 52 harnesses has only one instance.

Moreover we consider that there are 10 possibilities of gauge available per cables for all the instances and cases.

The first case, the simplest, contains 3 harnesses. It is constituted of 9 cables crossing 9 branches and grouping together 18 wires realizing 6 links. The size of search space of this case is $10^{9}$.

The second case contains 8 harnesses. It is constituted of 25 cables crossing 40 branches and grouping together 50 wires realizing 22 links. The size of search space of this case is $10^{25}$.

The third case contains 52 harnesses and represents an ATA (Air Transport Association). It is constituted of 404 cables crossing 406 branches and grouping together 643 wires realizing 200 links. The size of search space of this case is $10^{404}$.

The size of these search spaces are huge but it is possi- ble to reduce them by eliminating the impossible values determined by experts.

\subsection{Results}

With the Smart Harness Optimizer tool, for the 3-harnesses case and according to the instances, the resolution, with 153 agents, lasts between 1600 and 4700 milliseconds and requires between 60 and 160 cycles.

For the 8-harnesses case and according to the instances, the resolution, with 425 agents, lasts between 2100 and 4700 milliseconds and requires between 90 and 200 cycles. For the 52 harnesses case, the resolution, with 5548 agents, lasts about $2 \mathrm{~min}$ in 754 cycles. Besides the optimized weight, the tool shows each element violating a constraint and its characteristics.

All these cases were also tested by Labinal/Safran Engineering Services, the expert company that provides us the test cases. The used tool first reduces the search space (according to an experiment plan) and then finds the optimal solution. This enables to verify the relevance of solutions obtained with the Smart Harness Optimizer Tool.

\subsection{Analysis}

The main advantage with this method is the significant saving in time, for problems relatively big (the 52 harnesses). With the Smart Harness Optimizer tool, we found a solution in a few minutes, while with classical approaches it lasts several hours. We underline that the 52 harnesses is an ATA and in a plane there may be more than 10 ATA. Next experimentations could show the pertinence of our tool for problems having more than 52 harnesses. However we mention that for smaller cases ( 3 and 8 harnesses) the results are quite similar, even better for the classical approaches than with our developed tool concerning the 3 harnesses.

The second advantage is that our tool enables a fast adaptation in a real time to take into account dynamical changes and disruptions during the resolution time or once a solution is provided. This is particularly interesting when an engineer needs to change a value to make tests and comparisons. For instance he may decide to block a gauge value, or to change another one. Once this modification is applied, the resolution process does not start again from the beginning, but from the current solution, i.e. from the current computed values of variables. Only the neighborhood 


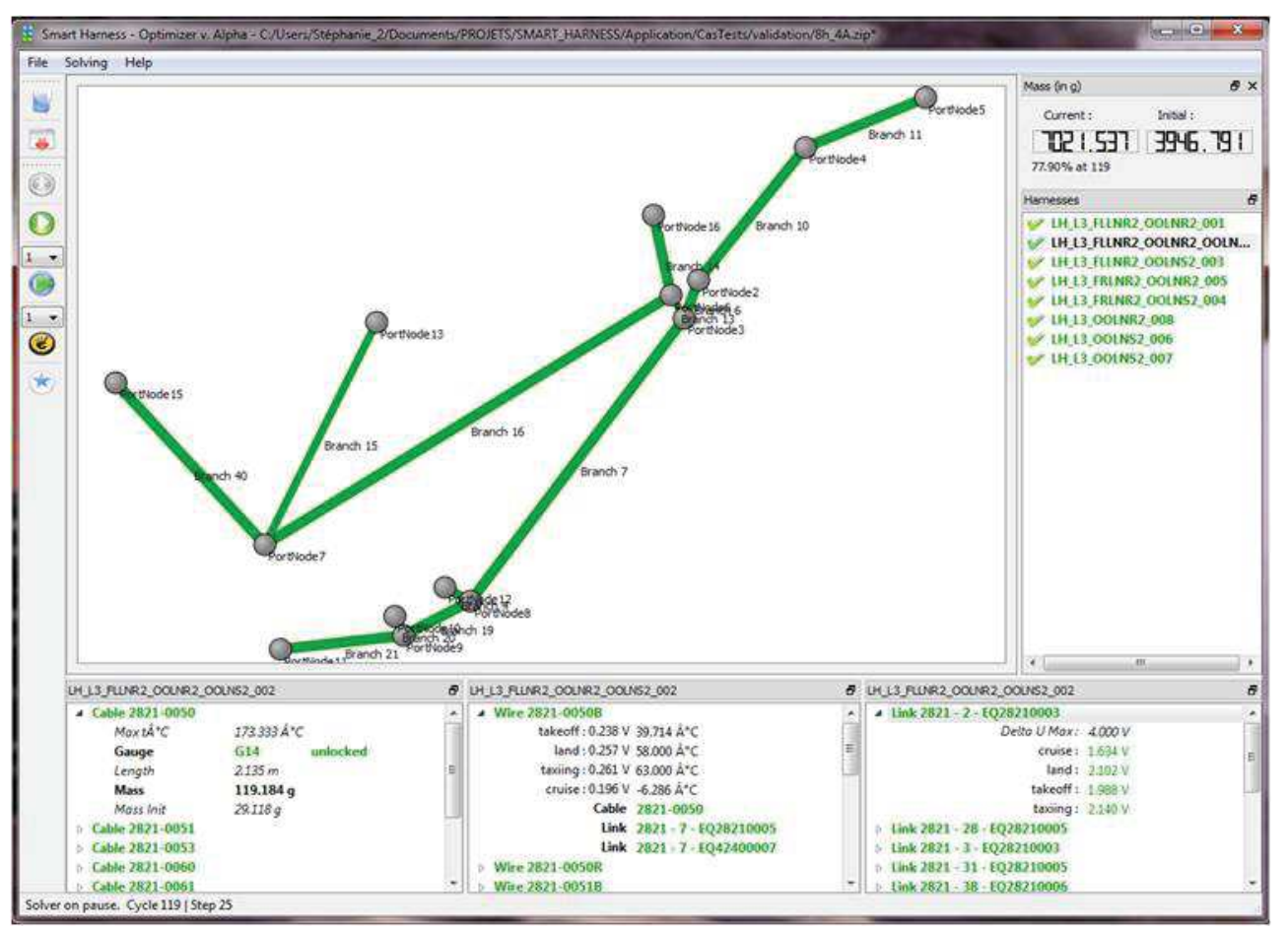

Figure 3: The Interface of the Smart Harness Optimizer.

of the agent whose value is modified is concerned and adapts itself to this new configuration. In other words the initiator agent of the modification propagates around its neighborhood change to other agents. This also leads to obtain new solutions in a quite short time.

\section{CONCLUSIONS}

This paper addresses the problem of weight optimization of aircraft harnesses that is multiconstrained and NP-complete. We deduce that considering the growing complexity of current applications, the multi-agent systems enable to get systems being robust, flexible and able to quickly adapt to the environment dynamic, thanks to the computation distribution and the control decentralization. Nevertheless these mechanisms require the implementation of local interactions between agents enabling them to coordinate locally their actions in order to produce a solution to the global level. In the resolution techniques using these mechanisms, we note that cooperation is a fundamental notion that rules the interactions and enhances the quality of obtained solutions.

We have developed a platform to solve it using the AMAS theory. This tool enables the harness designer i) to improve the sizing of harnesses by optimizing the diameter of wires, ii) to focus on elements that do not satisfy constraints and iii) to obtain the solution in a relatively short time. The harness weight thus optimized enables to reduce the operation costs of aircrafts.

This work is a first step but it offers numerous perspectives for industrials. By improving and enriching this software, this tool may help designers to reconfigure harnesses by inverting or changing cables from their harness. For instance if one cable poses problem because of constraints imposed on its harness, moving it to a new harness may decrease its constraints as its nearby environment has changed.

Going one step further, the tool could help designers to co-design harnesses. This co-design may assist them to specify in real time the most appropriate characteristics and make designers save design time by avoiding going back and forth between services. Going one more step further, this kind of tool could help in routing harnesses within the aircraft structure, by choosing the most appropriate way and it could also be coupled with the assignment of cables within harnesses.

Considering the performances of the operational tool, we think that a commercial software may help designers to the co-design of harnesses. This codesign may assist them to specify in real time the most appropriate characteristics like voltage drop. 


\section{ACKNOWLEDGEMENTS}

This work was realized within the French national project 'Smart Harness'. This project is cofunded by the 'Fond Unique Interministériel' and 'Région MidiPyrénées' and labeled by the pole of competitiveness Aerospace Valley. Upetec and Irit are specifically involved in the smart harness optimizer work package, in collaboration with the Labinal/Safran Engineering Services Company.

\section{REFERENCES}

Capera, D., Georgé, J., Gleizes, M., and Glize, P. (2003). The amas theory for complex problem solving based on self-organizing cooperative agents. In TAPOCS Workshop at 12th IEEE WETICE, pages 383-388.

Dorigo, M. and Stützle, T. (2004). Ant Colony Optimization. MIT Press.

Glize, P. (2001). L'Adaptation des Systèmes à Fonctionnalité Emergente par Auto-Organisation Coopérative. Habilitation à diriger des recherches, Université Paul Sabatier, Toulouse, France.

Glover, F. and Laguna, M. (1997). Tabu Search. Kluwer.

Hirayama, K. and Yokoo, M. (2005). The distributed breakout algorithms. In Artificial Intelligence, volume 161(1-2)), pages 671-680.

Holland, J. (1993). Adaptation in Natural and Artificial Systems. MIT Press.

Kennedy, J. and Eberhart, R. (2001). Swarm Intelligence. Morgan Kaufmann.

Kirkpatrick, S., Gellat, C., and Vecchi, M. (1983). Optimization by simulated annealing. In Science, volume 220(4598), pages 671-680.

Liu, J., Jing, H., and Tang, Y. (2002). Multi-agent oriented constraint satisfaction. In Artificial Intelligence, volume 136(1), pages 101-144.

Talbi, E. (2009). Metaheuristics: from design to implementation. Wiley.

Welcomme, J., Gleizes, M., and Redon, R. (2009). Selforganising multi-agent system managing complex system design application to conceptual aircraft design. In International Transactions on Systems Science and Applications, Systemics and Informatics World Network (SIWN), Special issue : Self-organized Networked Systems, volume 5(3), pages 208-221.

Yokoo, M., Durfee, E., and Kubawara, K. (1998). The distributed constraint satisfaction problem : Formalization and algorithms. In IEEE Transactions on Knowledge and Data Engineering, volume 10, pages 673685. 